\title{
Ultrasound-guided Percutaneous Trucut Needle Biopsy for Musculoskeletal Tumours
}

\author{
A Sallehuddin, MS Ortho (UM), A Saw, FRCS (Edin), J George*, FRCR, S Sengupta, FRCS (Edin) \\ Department of Orthopaedic Surgery, University Malaya Medical Centre, Malaysia \\ * Department of Biomedical Imaging, University Malaya Medical Centre, Malaysia
}

\begin{abstract}
Purpose: To evaluate the usefulness of ultrasound guidance in percutaneous needle biopsy for musculoskeletal tumours.

Methods: Forty-five consecutive patients underwent ultrasound-guided needle biopsy. An additional group of 50 patients who underwent needle biopsy without ultrasound guidance was retrospectively selected as historical control. The sample was considered adequate when a diagnosis can be made, and diagnostic when the diagnosis is similar to the final report based on the excised tumour.
\end{abstract}

Results: Adequacy of the biopsy samples was $84 \%$ in ultrasound-guided group as compared $76 \%$ in the group with no ultrasound guidance. Diagnostic accuracy was $64 \%$ in the ultrasound-guided group and $52 \%$ in the group without ultrasound guidance. Both of these differences were not statistically significant.

Conclusions: Ultrasound guidance did not provide a significant advantage in the biopsy of musculoskeletal tumours. Diagnostic accuracy seems to improve with the use of larger 14 gauge biopsy needle but further evaluation is necessary.

\section{Key Words:}

Ultrasound, Core needle biopsy, Soft tissue neoplasm, Diagnosis

\section{INTRODUCTION}

Musculoskeletal tumour is relatively uncommon and an accurate ratio of benign to malignant lesions is difficult to determine. Definitive diagnosis of the tumour is generally dependent on histopathological interpretation. Open biopsy remains the gold standard because it provides more tissue sample for examination compared to the less invasive needle biopsy. Some clinicians attempt to improve the diagnostic outcome of needle biopsy with the use of imaging techniques like image intensifiers or computerized tomography (CT scan).
High-resolution real time ultrasound allows for visualization of tissues of varying echogenicity and may provide guidance for the placement of biopsy needle. We conducted this study to determine the efficacy of ultrasound-guided needle biopsy for musculoskeletal tumour.

\section{MATERIALS AND METHODS}

Patients older than 12 years old and diagnosed with musculoskeletal (soft tissue and/or bone) tumour between February 2005 and September 2006 were considered for the study. Forty-five study participants underwent percutaneous biopsy utilizing a Trucut needle under the guidance of ultrasound. Those with intramedullary tumours with intact cortical bone that required a trephine type of biopsy needle were excluded, as were patients with bleeding disorder or those who had previous biopsy of the same lesion. The ethical committee of the hospital approved the study and written consent was obtained from all patients.

Fifty consecutive cases of musculoskeletal tumours, (treated with percutaneous biopsy followed by definitive surgical resection) at the same institution between 1998 and 2000 were selected as historical controls. We traced the histopathological report of the first needle biopsy and the final pathological report following definitive surgical treatment.

The biopsy sample was considered adequate if a provisional diagnosis could be made from the first tissue sample. If a second needle biopsy or an open biopsy was required, the first sample was considered inadequate. The biopsy sample was considered diagnostic when the provisional diagnosis corresponds with diagnosis made following examination of the tumour tissue removed by definitive surgery.

\section{Needle biopsy under ultrasound guidance}

Colour Doppler ultrasound was used to evaluate the vascularity of the tumour and to locate major vessels located close to the biopsy site ${ }^{10}$. The ultrasound machine used was a LOGIQ 700MR (GE Medical Systems Milwaukee, WI) equipped with a MI12L 6-14 MHz and a 546L 3-6 MHz transducer. 
Table I: Final diagnosis for the ultrasound-guided group

\begin{tabular}{|c|c|}
\hline Final Diagnosis & Number \\
\hline Lipoma & 7 \\
\hline Metastases & 4 \\
\hline Fibromatoses & 3 \\
\hline Osteosarcoma & 3 \\
\hline Schwannoma & 2 \\
\hline Neurofibroma & 2 \\
\hline Malignant Fibrous Histiocytoma & 2 \\
\hline Chondrosarcoma & 2 \\
\hline PVNS (pigmented villonodular synovitis) & 1 \\
\hline Hemangioma & 1 \\
\hline Tuberculosis & 1 \\
\hline Sebaceous Cyst & 1 \\
\hline Synovial Chondromatosis & 1 \\
\hline Epidermal Cyst & 1 \\
\hline Chondroma & 1 \\
\hline Atypical Myofibroblastic Tumour & 1 \\
\hline Fibrous Dysplasia & 1 \\
\hline Ewings Sarcoma & 1 \\
\hline Synovial Sarcoma & 1 \\
\hline Osteomyelitis & 1 \\
\hline Myxoid Sarcoma & 1 \\
\hline Non Hodgkins Lymphoma & 1 \\
\hline Carbuncle & 1 \\
\hline Pseudogout & 1 \\
\hline Granular Cell Tumour & 1 \\
\hline Spindle Cell Mesenchymal Tumour & 1 \\
\hline Rhabdomyosarcoma & 1 \\
\hline Infective Arthritis & 1 \\
\hline TOTAL & 45 \\
\hline
\end{tabular}

Table II: Final diagnosis for the Nonultrasound-guide group

\begin{tabular}{|c|c|}
\hline Final Diagnoses & No \\
\hline Osteosarcoma & 13 \\
\hline Giant Cell Tumour & 12 \\
\hline Liposarcoma & 5 \\
\hline Chondrosarcoma & 2 \\
\hline Ewings Sarcoma & 2 \\
\hline Fibrous Dysplasia & 2 \\
\hline Angiosarcoma & 1 \\
\hline Non Hodgkins Lymphoma & 1 \\
\hline Fibrosarcoma & 1 \\
\hline Chondromyxoid Fibroma & 1 \\
\hline Osteochondroma & 1 \\
\hline Lipoma & 1 \\
\hline Osteoblastoma & 1 \\
\hline Aneurysmal Bone Cyst & 1 \\
\hline Malignant Fibrous Histiocytoma & 1 \\
\hline Tuberculosis & 1 \\
\hline P.N.E.T. & 1 \\
\hline Rhabdomyosarcoma & 1 \\
\hline Hemangioma & 1 \\
\hline Chordoma & 1 \\
\hline TOTAL & 50 \\
\hline
\end{tabular}




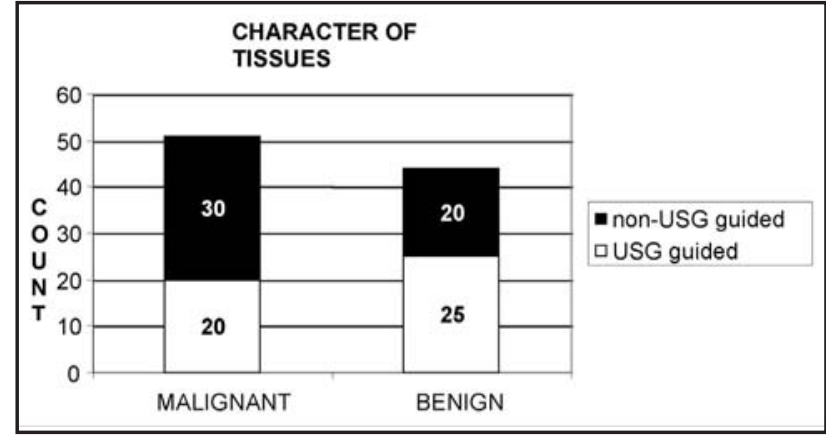

Fig. 1: Character of tissue between the ultrasound-guided and the nonultrasound-guided groups.

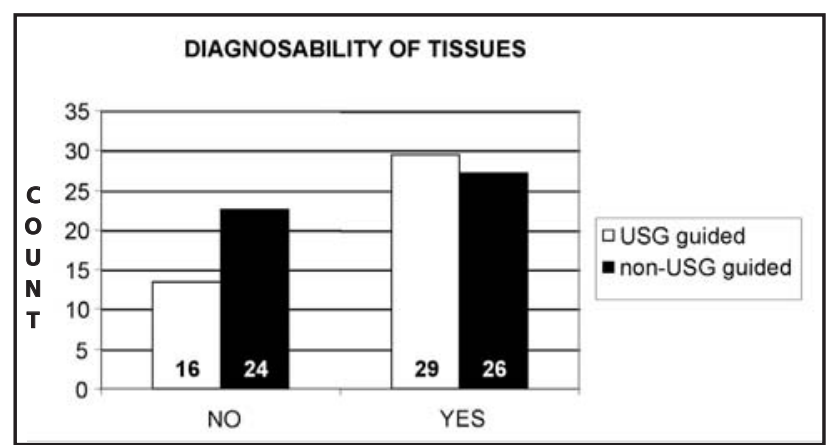

Fig. 3: Comparison of diagnosability between the Ultrasoundguided and the Nonultrasound-guided Groups.

The skin was sterilized with povidone iodine or hexachloride solution. About 5 to $10 \mathrm{ml}$ of $1 \%$ lignocaine was infiltrated to provide local anaesthesia. The probe was enveloped in a sterile plastic bag and examination performed. The cutting needle was inserted under direct visualization so that areas of tissue necrosis were avoided. Tissue specimens were obtained from the interface area of the tumour. Sampling of various parts of the tumour was then performed using a 16 gauge Temno automated Trucut biopsy needle. Based on the results of the first 29 cases, a change was made for the last 16 cases in that larger 14 gauge needles was used. For each biopsy, 2 to 4 cores were obtained depending on the quality of the tissue obtained and the heterogeneity of the tumour on ultrasound examination.

\section{RESULTS}

In the ultrasound-guided biopsy group ( 45 cases), there were 20 (44.4\%) patients with malignant lesions and 25 (55.6\%) patients with benign lesions. The most common pathology was lipoma, followed by soft tissue metastasis, fibromatosis and osteosarcoma (Table I). In contrast, we found that for the historical control group (50 cases), the most common diagnosis was osteosarcoma, followed by giant cell tumour and liposarcoma (Table II); thirty (60\%) patients had malignant lesions and $20(40 \%)$ patients had benign lesions in this group (Figure 1).

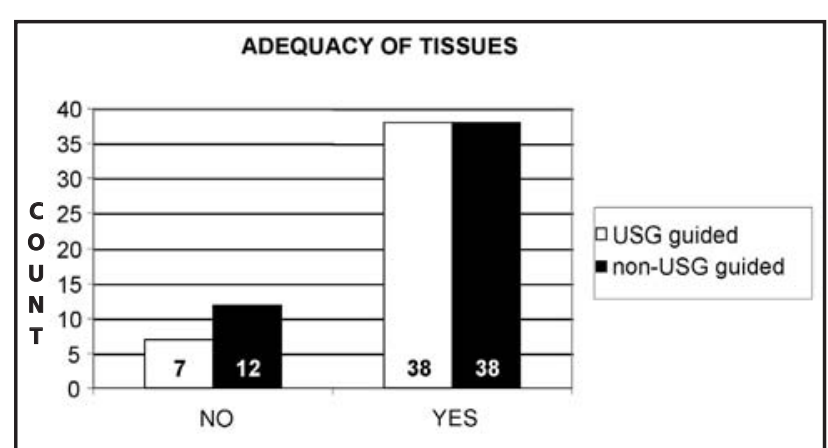

Fig. 2: Comparison of adequacy of tissue diagnosis between the ultrasound-guided and the nonultrasound-guided groups. $p$ value 1.056 using the Pearson chi square test.

In the ultrasound-guided group 38 out of 45 (84\%) biopsy procedures provided adequate tissue for interpretation, while for the historical control group 38 out of $50(76 \%)$ biopsy procedures provided adequate samples for interpretation. The difference was however not statistically significant (Figure 2).

In the ultrasound-guided group 29 out of 45 (64\%) biopsy tissues were considered diagnostic compared to nonultrasound-guided group where 26 out of $50(52 \%)$ biopsy samples were diagnostic, also not a statistically significant difference (Figure 3).

Decision to use a larger biopsy needle was made following early review of the results since there was not an obvious difference between the ultrasound-guided and nonultrasound-guided groups. For the last 16 cases where a 14 gauge needles were used, we managed to obtain adequate tissue for interpretation in all cases $(100 \%)$ and the samples were diagnostic in 13 cases $(81 \%)$. Due to the limited number of cases with the larger gauge needles, we were not able to conclude that these differences were significant.

\section{DISCUSSION}

We were not able to discern any advantage in the use of ultrasound for diagnosis of musculoskeletal tissue tumour. Ultrasound may not be able to provide useful guidance for diagnostic purposes where more diagnostic tissue can be obtained via other methods. On the other hand, the use of ultrasound guidance may improve the safety of the biopsy procedure. With the use of colour Doppler ${ }^{15}$, location of major vessels adjacent to the tumour can be identified and injuries avoided.

Ahrar et al reported that most malignant primary bone lesions have a substantial extra osseous component that can be identified by ultrasound and then biopsied ${ }^{14}$. Sampling purely the osseous components of lesions can yield inconclusive diagnoses or limited samples of crushed bone fragments that might not be adequate for diagnosis. In 
advanced cases these advantages of ultrasound-guided biopsy may not be obvious.

Larger biopsy needle used in the later stage of this study showed what appears to be a significant increase in the accuracy of the biopsy technique; however, the design of this study does not allow us to investigate whether it was the larger bore needle or the ultrasound guidance that contributed to this trend. This issue should be addressed in a follow-up study.

\section{CONCLUSION}

In the biopsy of musculoskeletal tumours, ultrasound guidance did not provide significant advantage in terms adequacy and diagnostic accuracy of the biopsy specimen. Further research is needed to investigate potential benefits of using ultrasound for biopsy with a larger gauge needle. 


\section{REFERENCES}

1. Ahrar K, Himmerich JU, Cynthia EH, Raymond AK, Wallace MJ, Gupta S. Percutaneous ultrasound-guided biopsy in the definitive diagnosis of osteosarcomas. J Vasc Interv Radiol 2004; 15: 1329-33.

2. Ball AB, Fischer C, Pittam M, Watkins RM, Westbury G. Diagnosis of soft tissue tumours by Tru - cut biopsy. Br J Surg 1990; 77: 756-8.

3. Chiou HJ, Chou YH, Wu JJ. The role of high resolution ultrasound in management of calcific tendonitis of the rotator cuff. Ultrasound Med Biol 2001; 27(6): 735-43.

4. Chiou HJ, Chou YH, Wu JJ. Alternative and effective treatment of shoulder ganglion cyst: ultrasonographically guided aspiration. J Ultrasound Med 1999; 18: 531-5.

5. Christensen RA, Van Sonnenberg E, Casola G, Wittich GR. Interventional ultrasound in the musculoskeletal system. Radiol Clinic North Am 1998; 26: 145-56.

6. Farin PU, Rasanen H, Jaroma H, Harju A. Rotator cuff calcifications: treatment with ultrasound -guided technique percutaneous needle aspiration and lavage. Skeletal Radiol 1996; 25(6): 551-4.

7. Fornage BD. Soft tissue masses: The underutilization of sonography. Seminars in musculoskeletal radiology. 1999; 3: 115-33.

8. Frates MC, Benson CB, Doubilet PM, Edmund SC, Ellen M. Can color Doppler aid in the prediction of malignancy of thyroid nodules? J Ultrasound Med 2003; 22: 127-31.

9. Henry J, Carole J, Michael AS. (1996). Hazards of biopsy revisited. J Bone Joint Surg (A) 78: 656-63.

10. Jesus Z, Michael EB, Nelson, GO, John L, Michael AC. Percutaneous Needle Biopsy of Soft Tissue Tumors Guided by Ultrasound and Computed Tomography. Skeletal Radiol 1990; 9: 33-6.

11. Keohan ML, Taub RN. Chemotheraphy for advanced sarcoma: therapeutic decisions and modalities. Semin Oncol 1997; $24: 572-9$.

12. Liu JC, Chiou HJ, Chen WM, Chou YH, Chen TH, Chen W, Chueh CY, See YC, Chang CY. Sonographically Guided Core Needle Biopsy of Soft Tissue Neoplasms. J Clin Ultrasound 2004; 32: 294-8.

13. Mankin HJ, Lange TA, Spanier SS. The hazards of biopsy in patients with malignant primary bone and soft tissue tumors. $J$ Bone Joint Surg 1982; 64-A: 1121-7.

14. Rubens DJ, Fultz PJ, Gottlieb RH, Rubin SJ. Effective ultrasonographically guided intervention for diagnosis of musculoskeletal lesions. J Ultrasound Med 1997; 16: 831-42.

15. Sofka CM, Collins AJ, Adler RS. Use of ultrasonographic guidance in interventional musculoskeletal procedures: a review from a single instituition. J Ultrasound Med 2001; 20: 21-6.

16. Yeow KM, Tan CF, Chen JS, Hsueh C. Diagnostic sensitivity of ultrasound-guided needle biopsy in soft tissue masses about superficial bone lesions. J Ultrasound Med 2000; 19: 849-55. 